\title{
Switch Operating Mechanism Box Integrated Wireless Temperature Monitoring Terminal
}

\author{
Wang zhenhao ${ }^{1, a}$, Chen hongtao ${ }^{2, \mathrm{~b}}$, Zhao jianming ${ }^{2, \mathrm{c}}$, Meng xiangchen $^{2, \mathrm{~d}}$ \\ 1. Northeast Dianli University, Jilin Jilin 132012, China \\ 2. State Grid Jilin Electric Power Company Limited Songyuan Power Supply Company, Songyuan, Jilin Songyuan 138000, China
}

\begin{abstract}
Switch operating mechanism box integrated wireless temperature monitoring terminal is designed and the sensor circuit, the heater current detection circuit, a wireless transceiver circuit, the control circuit are developed in the present invention.So the mechanism box temperature change can be monitored and operated in real time, heating equipment can be controlled according to the operating temperature, monitoring date can be transmitted to the analysis and management system through wireless. Design of the monitoring terminal has got through test of actual field operation.
\end{abstract}

\section{Introduction}

Many outdoor operating equipment of transformer substation are influenced by temperature seriously, operating conditions of high voltage switches and other equipment, especially temperature is an important indicator of safe operation of equipment. In recent years, the requirement of reliability in operation and monitoring on electrical equipment has been increasingly high. Temperature of high-voltage switch operating mechanism and heat tapes affect the proper operation of the switch directly(especially in winter of the northern region), most of traditional temperature control device consist of simple U-type electric heating tube, AC contactor, thermometer and without a complete monitoring and control system which can't realize the switching work environment in time, there exists big hidden faults.

After the substation unattended, if the heating device does not work can't be found in time which would be a treat to the safe operation of equipment.

The invention "Switch operating mechanism box integrated wireless temperature monitoring terminal" can monitor the temperature change of high-voltage switch operating mechanism box by radio frequency networking, and the measured data will be transmitted to the remote monitoring host in station via radio frequency communication. The control circuit control heating device according to the parameters set by the operating temperature, which make temperature in the mechanism box is maintained at a certain range. Heater using PTC ceramic materials and axial fans with energy-efficient security features, is conducive to the stable operation of high voltage switch.

\section{Integrated switch mechanism box wireless monitoring terminal program}

Monitoring terminal consist of microcontroller, temperature sensor, control circuit, wireless radio communication circuit, heater current detection circuit, power supply circuit and other components. Digital data conversed by temperature sensor is sent to CPU through I2C bus, processed signals sent to $\mathrm{AD}$ conversion then are sent to CPU for data processing, and the measured data can be sent to the remote data acquisition repeater by radio frequency circuit. The control circuit is controlled according to parameters set by the switch operating temperature, so that the temperature inside the mechanism box can be maintained in a certain range. Heater current detection circuit through the three-phase CT current detector to determine if the heater is working normally, and it's easy to repair. The overall structure is shown in Figure 1.

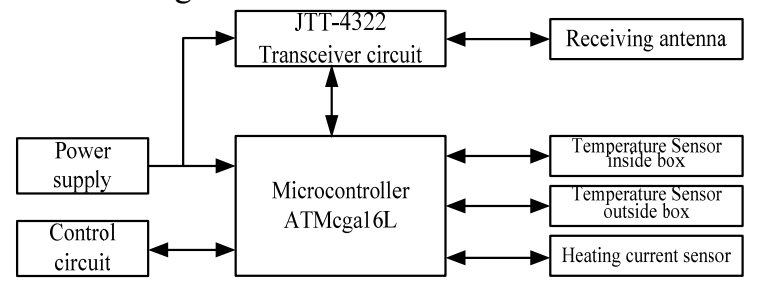

Figure 1. The overall structure of the monitoring terminal

\subsection{Microcontroller}

Microcontroller using Atmel's AVR single chip microcomputer ATMEGA16L, with a high cost performance. ATMEGA16L's working voltage is 2.7-5,5V,under the condition of $1 \mathrm{MHz}, 3.3 \mathrm{~V}$, the normal working current is about $200 \mu \mathrm{A}$, the free working current is $0.35 \mathrm{~mA}$ 。Shown as Figure 2 .

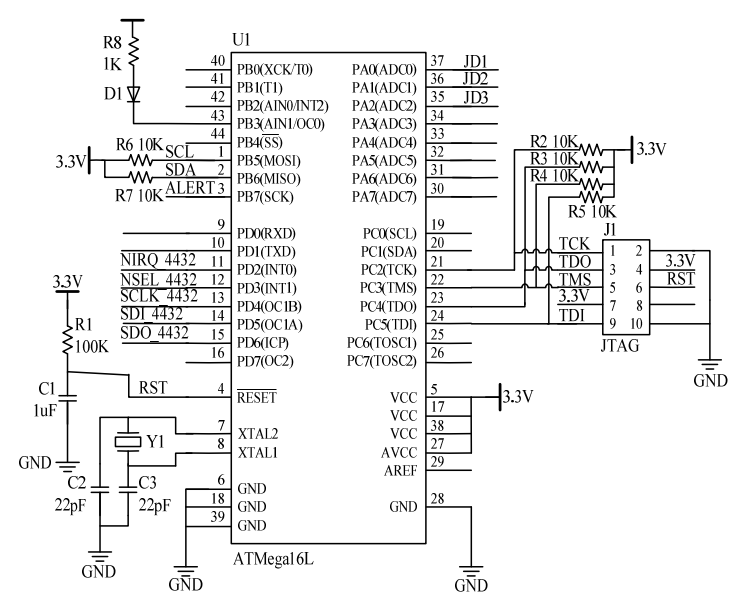

Figure 2. Microcontroller circuit 


\subsection{Temperature and humidity sensor}

Temperature and humidity sensor using type I2C bus digital temperature and humidity sensor SHT10 produced by Sensirion. The sensor includes a capacitive polymer humidity measuring sensor, a temperature measurement made by bandgap material, achieve seamless connectivity with 14-bit $\mathrm{AD}$ converter and a serial interface circuit on the same chip. The measurement range of SHT10 is from $-40 \sim+125^{\circ} \mathrm{C}$, and have the accuracy of $\pm 0.5^{\circ} \mathrm{C}$; humidity accuracy of $\pm 4.5 \%$ RH. Shown as Figure 3 .

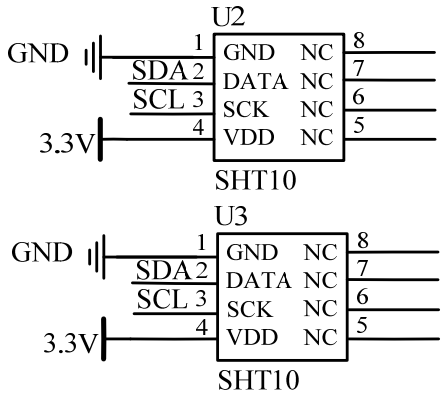

Figure 3. Temperature and humidity sensor circuit

\subsection{Heater current detection circuit}

Monitoring terminal can monitor whether the heater device is working properly, access current transformer on the heater power (considering three-level heating), signal via a resistor into a voltage signal, after finishing input to the emitter follower then sent to the AD digital-analog conversion circuit, and conversed to digital which is sent to CPU to calculate, so weather the heater is working properly and need to maintain can be judged.

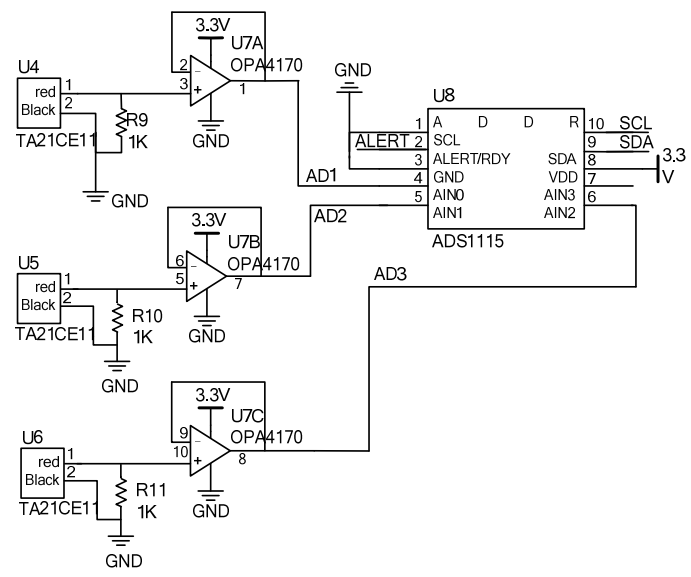

Figure 4. Heater current detection circuit

\subsection{Wireless transceiver circuit}

Wireless transceiver circuit based on high performance radio frequency chip Si4432 works in $433 / 470 / 915 \mathrm{MHz}$ general ISM band with a modulator and demodulator, data can be sent with automatic packaging and accepted with automatic address match, automatic CRC check, after sending and receiving end, it's NIRQ interrupt pin will be set high level automatically to indicate transmit or receive completed, Si4432 controller provides a SPI interface to the applied controller, the speed is determined by controller itself, so the programming is very convenient. Its power consumption is very low, when it emit at the output power of $20 \mathrm{dBm}$ the current is $85 \mathrm{~mA}$ and under reception mode the current is only $15 \mathrm{~mA}, 2.5 \mu \mathrm{A}$ under standby mode. Si4432 has four operating modes, respectively, down and SPI programming mode, standby and SPI programming mode, the reception mode, and the transmission mode. Its work mode switch achieve by configuration register 07H, as shown in Figure 5.

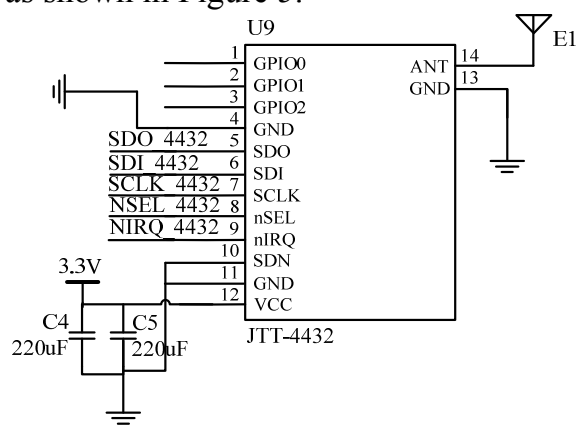

Figure 5. Radio frequency communication circuit

\subsection{Control circuit}

Monitoring terminal monitors the temperature change inside switch mechanism box in real-time, when the temperature is below the set temperature, the heater begins, heater working(three level heated) started by the relay work which is drived by integrated drive circuit ULN2003, when it is higher than the set temperature, stop the heat working. Shown as Figure 6.

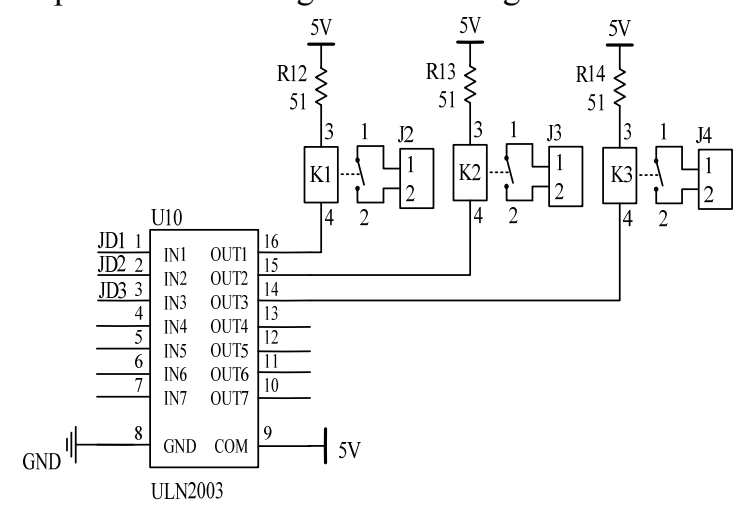

Figure 6. Control circuit

\section{Field operation}

Installation and commissioning of monitoring terminal has been set in Changling $220 \mathrm{kV}$ substation and running. Monitoring terminal on field and monitoring data are shown in Figure 7. 


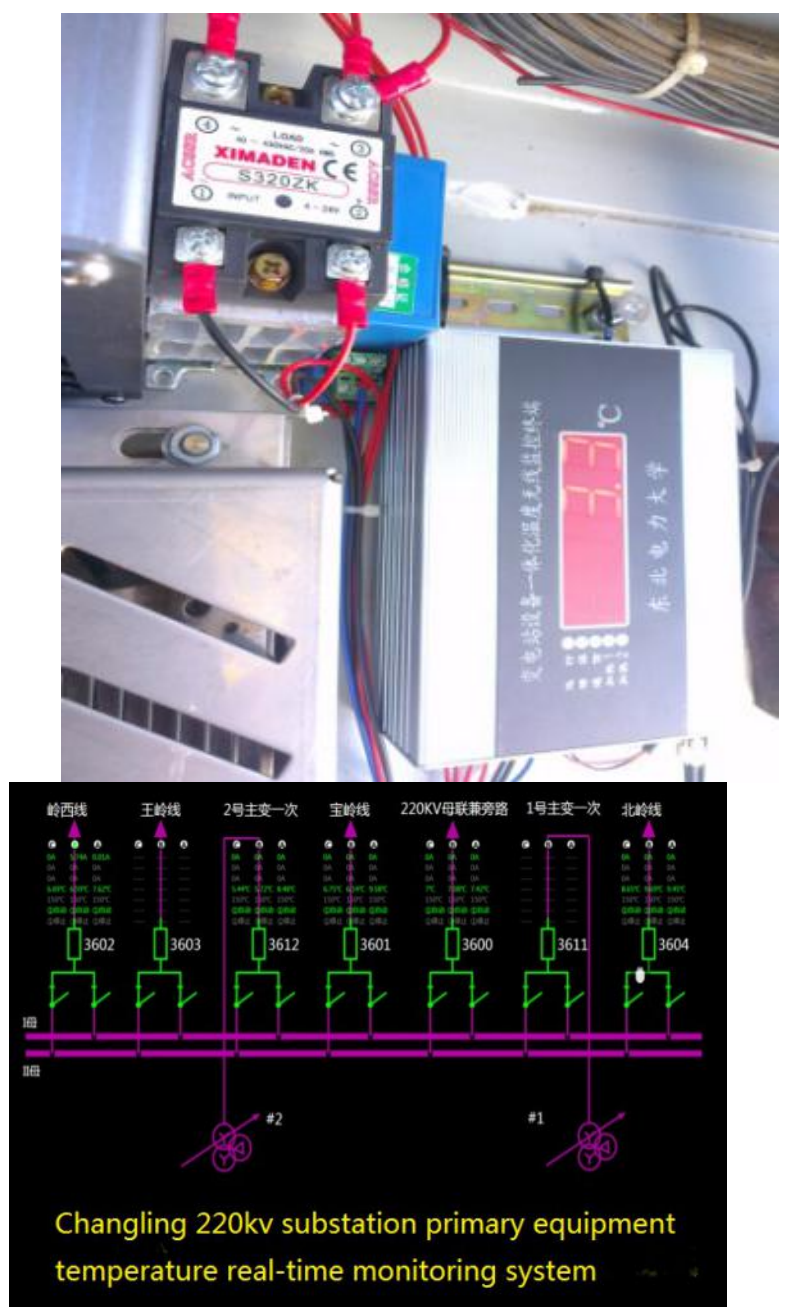

Figure 7. Field monitoring device and monitoring data

\section{Concluding remarks}

This design of the switch operating mechanism box integration of wireless temperature monitoring terminal, with the test of laboratory and the field, every indicator has reached the design requirements, real-time monitoring the temperature change inside mechanism box, using PTC ceramic materials and axial fans which have energy-efficient security features, is conducive to stable operation of high voltage switch.

\section{Reference}

[1] HE Yi, ZHOU Yan, CAO Weiguo, et al. Application research of optic fiber sensor to the on-line HV switchgear monitoring system. East China Electric Power, 39, 9(2011)

[2] WU Gang, LI Jin. Application of optical fiber in temperature monitoring of the contacts of switch cabinet. High Voltage Engineering, 32, 2(2006)

[3] YANG Fan. The research of wireless temperature measurment system for the high-voltage switchgear Wuhan:Huazhong University of Science and Technology, 2011

[4] WU Guangrong, ZHANG Jianxiong. A kind of temperature and humidity monitoring system for high pressure switch cabinet based on ZigBee technology. Modern Electronics Technique, 31, 20 (2008).

[5] ZHOU Wei,ZHU Qiang, XU Min jie, et al. Development of Infrared Monitoring System for High Voltage Switch Cabinet. Electrical Measurement \& Instrumentation, 50, 12 (2013)

[6] GONG Xian feng, YI Honggang, WANG Chang song, et al . Research on Temperature Monitoring of Isolators in $\mathrm{HV}$ Switchgear. Proceedings of the CSEE, 26, 1(2006) 